\title{
The need for integrative oncology and a remodeling of evidence-based medicine
}

\begin{abstract}
Abbreviations: CAM, complementary alternative medicine; EBM, evidence-based medicine; RCT, randomized controlled trial; TCM, traditional chinese medicine; PBM, patient-based or centered medicine; PCC, patient-centered communication; VEGF, vascular endothelial growth factor; mTOR, mammalian target of rapamycin
\end{abstract}

\section{Editorial}

By reviewing various published research articles it is known that over $70 \%$ of women with breast cancer. ${ }^{1,2}$ as well as significant percentage of other cancer patients, ${ }^{3,4}$ use some form of complementary alternative medicine (CAM) in conjunction with conventional bio-medical oncology. CAM use (both self-medication with products and visits to CAM practitioners) increased significantly from 1998 to $2008 .{ }^{5}$ In another study more than $80 \%$ of all women with breast cancer report using CAM ( $41 \%$ in a specific attempt to management their breast cancer), CAM use can no longer be regarded as an "alternative" or unusual approach to managing breast cancer. ${ }^{6}$. Patients use CAM because they want to feel they have some control over their cancer and improve quality of life, ${ }^{7,8}$ although there is not always ample evidence or conflicting evidence to support its use. ${ }^{9,10}$

There are not clear numbers regarding the specific use of botanical medicine in the CAM statistics but Hyodo et al.. ${ }^{11}$ reported that herbs and other dietary supplements are used by substantial numbers of cancer patients in Japan- $44.6 \%$ of 3,100 patients. A high proportion of advanced-stage breast cancer patients used CAM. Discussion with doctors was high for ingested products. ${ }^{12}$. In spite of the fact that there are conflicting attitudes in CAM use, there are not, nor there likely be considerable numbers of randomized controlled trials (RCTs) or Phase IV clinical trials on any of the botanical medicines due to real financial limitations. ${ }^{13}$

\section{Discussion}

Most botanicals used in conjunction with bio-medical cancer treatment are safe and effective, ${ }^{14-16}$ and there is good research on drug-herb interactions as well. ${ }^{17-20}$ There is a long history of use as well as extensive use in hospitals in many countries, although primarily in China. $98 \%$ of patients had used at least one form of CAM therapy after diagnosis of breast cancer in Shanghai. The most popular CAM modality was traditional Chinese medicine $(86.7 \%)$, followed by the use of supplements $(84.8 \%)$, physical exercises $(65.5 \%)$, and support group attendance (16.6\%). Chinese herbal medicine (CHM) was used by $86.4 \%$ of patients, while acupuncture was used only by $4.9 \%$ of patients. ${ }^{21,22}$ Some oncologists seek to limit patient use of these substances, even though there is little or no published evidence as to their contraindications, ${ }^{23}$ but patient continue to use them anyway. While academic research using RCTs are the bedrock of evidencebased medicine, the whole area of complementary oncology is peripheral to mainstream paradigms, yet patient will and do seek them out whether their oncologist knows that they do so. ${ }^{24}$

Centers such as Memorial Sloan-Kettering, Dana-Farber, and University of California at several sites, M.D. Anderson, and many more now have programs that clinically integrate conventional and complementary medicine. They also conduct research on
Volume I Issue I - 2015

\author{
Daniel Weber \\ Visiting Professor, Tianjin University, Australia
}

Correspondence: Daniel Weber, Consultant at National Cancer Institute, 74 Brandling St, Alexandria NSW 2015, Australia,Tel 61 2 9519 2233,Email drdweber@panaxea.com

Received: March 10, 2015 | Published: March 12, 2015

complementary therapies and on herbs and other supplements. By bringing these issues into mainstream practice and research-rejecting useless treatments, offering those of proven value, and systematically developing those that show promise is critical. ${ }^{25}$ It is possible to emulate Japan's regulatory efforts and ensure that the phenomenon described by Hyodo et al. ${ }^{11}$ can be established worldwide. This approach can be developed in the 'Western' world as well as it becomes a force for the greater well being of cancer patients. ${ }^{26}$

It is believed the use of botanicals in conjunction with bio-medical oncology practice is the wave of the future and needs to be codified and implemented in a formal fashion. Traditional Chinese Medicine is part of 'Systems Biology' approach and operates outside but alongside a conventional reductionist, Newtonian research paradigm. ${ }^{27}$ Systems Biology focuses on complex interactions within biological systems, using a holistic approach (holism instead of the more traditional reductionism) to biological and biomedical research. Metabolomics, genomics and oncogenomics, proteomics as well as the whole area of Omics research, ${ }^{28}$ needs to be applied to complementary oncology and we need to move medical science from its Newtonian/Modernism underpinnings to a holistic approach where other sciences have arrived. ${ }^{29}$ We need to deconstruct medicine.

Evidence-based medicine (EBM) claims to be based on 'evidence', rather than 'intuition' . ${ }^{30}$ However, EBM's fundamental distinction between quantitative 'evidence' and qualitative 'intuition' is not selfevident. The meaning of 'evidence' is unclear and no studies of quality exist to demonstrate the superiority of EBM in health care settings. ${ }^{31}$. EBM as currently conceptualized cannot accommodate concepts that resist quantitative analysis and therefore cannot logically differentiate human beings from complex machines. ${ }^{32,33}$

RCTs are considered the 'Gold-standard' in EBM but are impersonal in nature and fail to see the individual patient's presentation of their disease. The very word 'randomized and blinded' suggests this depersonalized approach. The multi factorial basis of many health outcomes is not addressed in EBM and thus the need for more sophisticated, multi dimensional, community-based designs. ${ }^{32}$ RCTs have limited capacity to assess such comprehensive initiatives. They also are limited in assessing aspects of quality. ${ }^{34}$ as well as performance measurement. ${ }^{35}$

Patient-based or centered medicine (PBM), also called holistic medicine sees uniqueness of patients, their individual needs and 
preferences, and their emotional status are easily neglected as relevant factors in decision-making. PBM has basically a humanistic, bio psychosocial perspective, combining ethical values on 'the ideal physician', with psychotherapeutic theories on facilitating patients' disclosure of real worries, and the negotiation on decision making. ${ }^{36}$ Part of PBM is patient centered communication (PCC), which helps practitioners provide care that is concordant with the patient's values, needs and preferences, and that allows patients to provide input and participate actively in decisions regarding their health and health care. ${ }^{37}$ Doctors increasingly regard a 'patient-centered' approach as crucial for the delivery of high quality care. Five conceptual dimensions in PBM are identified: bio psychosocial perspective; 'patient-as-person'; sharing power and responsibility; therapeutic alliance; and 'doctoras-person'. ${ }^{38}$ The implication is that in order to understand illness and alleviate suffering, medicine must first understand the personal meaning of illness for the patient. ${ }^{39}$

Research, where a systems approach is used means each patient is viewed as unique and their treatment is unique to their needs; we treat the patient not their disease. TCM and other traditional medicines are ancient systems medicine, where diagnosis by traditional means determined the protocol used and this is already highly quantified. Its limit is the lack of integration between the language used to understand the "Pattern" and current bio-medical diagnostic language but I believe this is now possible to bridge this gap. TCM concepts are sound but the language associated with these concepts need translating into a modern idiom.

Diagnostic techniques also need updating, using modern approaches to confirm traditional understandings of "Pattern". In TCM, "pattern" ( $\square$, pinyin: zhèng) refers to a "pattern of disharmony" or "functional disturbance" within the functional concept the TCM model of the body. ${ }^{40}$ It is not the disease itself but the underlying dynamics by which the disease arose. In the past two decades, studies in TCM Zheng have increased dramatically along with advances in medical technologies. Confronted with the large and increasing volume of TCM data, an urgent need emerges to explore these resources effectively using techniques of knowledge discovery in databases (KDD). ${ }^{41}$ Jiang et al. ${ }^{42}$ believe that effective data mining approach applications in the analysis of multiple extensively integrated databases (such as the TCM database SinoMed for TCM Zheng classification and the PubMed database for biomedicine) can supply new information in TCM Zheng research, including findings regarding the basic rules of Zheng distribution in certain diseases; the correlations between Zheng, disease, and herbal prescriptions; and the build-up of Zheng-Zheng and Zheng-disease correlation networks.

Research protocols themselves need re-evaluation and a need for finding ways to create a systems biology approach, which focuses on complex interactions within biological systems, using a holistic approach (holism instead of the more traditional reductionism). Current developments using circulating tumor cells, where individual medicinals can be matched to the patient's cancer cells is one way forward. Another is to look at and regulate various pathways such as VEGF, mTOR etc. rather the tumor type.

\section{Conclusion}

I believe the current EBM/RCT scientific approach has gone by its use-by-date and we need to revision; create a new, yet valid medical approach, which will inevitably benefit the patient, not just the medical industry. Yes, good integrative approaches to health-care will not only be effective but cheaper ${ }^{43}$ as it grows in usage. ${ }^{5}$ An Omics approach allows for individual patient treatment, which is a saving, both in results achieved and costs involved. ${ }^{44}$ The ZHENGOmics approach possesses and integrates the characteristics of both ZHENG and systems biology and may advance TCM to a new level. It would overcome the shortcomings of current methods for evaluating personal diagnosis, complex intervention, and patients' feelings. Through ZHENG-Omics study, personal medicine in TCM may emerge eventually and may change the current medical system. ${ }^{45}$ While the ZHENG/TCM approach to diagnostics might appear nonobjective, it does offer long-standing clinical and phenotypic-rich insights. With the current globalization of life sciences and the arrival of "Big Data" research and development, these two silos of medical lore are rapidly coalescing. The applications of multi-Omics strategies to TCM have begun to provide novel insights into the essence and molecular basis of TCM ZHENG. ${ }^{46}$

\section{Acknowledgments}

None.

\section{Conflicts of interest}

The authors declare there are no conflicts of interest related to the article.

\section{References}

1. Adler SR, Fosket JR. Disclosing complementary and alternative medicine use in the medical encounter:a qualitative study in women with breast cancer. J Fam Pract. 1999;48(6):453-458.

2. Ashikaga T, Bosompra K, Brien OP, et al. Use of complimentary and alternative medicine by breast cancer patients:prevalence, patterns and communication with physicians. Support Care Cancer. 2002;10(7):542548 .

3. Molassiotis A, Fernadez-Ortega P, Pud D, et al. Use of complementary and alternative medicine in cancer patients:a European survey. Ann Oncol. 2005;16(4):655-663.

4. Yates JS, Mustian KM, Morrow GR, et al. Prevalence of complementary and alternative medicine use in cancer patients during treatment. Support Care Cancer. 2005;13(10):806-811.

5. Nahin RL, Barnes PM, Stussman BJ, et al. Costs of Complementary and Alternative Medicine (CAM) and Frequency of Visits to CAM Practitioners. 2009;18:1-16.

6. Boon HS, Olatunde F, Zick SM. Trends in complementary/alternative medicine use by breast cancer survivors:Comparing survey data from 1998 and 2005. BMC Womens Health. 2007;7:4.

7. DiGianni LM, Garber JE, Winer EP. Complementary and Alternative Medicine Use Among Women With Breast Cancer. J Clin Oncol 2002;20(18 Suppl):34S-38S.

8. Henderson JW, Donatelle RJ. Complementary and alternative medicine use by women after completion of allopathic treatment for breast cancer. Altern Ther Health Med. 2004;10(1):52-57.

9. Balneaves LG, Truant TLO, Kelly M, et al. Bridging the gap:decisionmaking processes of women with breast cancer using complementary and alternative medicine (CAM). Support Care Cancer. 2007;15(8):973983.

10. Evans M, Shaw A, Thompson EA, et al. Decisions to use complementary and alternative medicine (CAM) by male cancer patients:informationseeking roles and types of evidence used. BMC Complement Altern Med. 2007;7:25. 
11. Hyodo I, Amano N, Eguchi K, et al. Nationwide survey on complementary and alternative medicine in cancer patients in Japan. J Clin Oncol 2005;23(12):2645-2654.

12. Shen J, Andersen R, Albert PS, et al. Use of complementary/alternative therapies by women with advanced-stage breast cancer. BMC Complement Altern Med. 2002;2:8

13. Sanson-Fisher RW, Bonevski B, Green LW, et al. Limitations of the randomized controlled trial in evaluating population-based health interventions. Am J Prev Med. 2007;33(2):155-161.

14. Shu $\mathrm{X}$, McCulloch $\mathrm{M}$, Xiao $\mathrm{H}$, et al. Chinese herbal medicine and chemotherapy in the treatment of hepatocellular carcinoma:a meta-analysis of randomized controlled trials. Integr Cancer Ther. 2005;4(3):219-29.

15. McCulloch M, See C, Shu XJ, et al. Astragalus-Based Chinese Herbs and Platinum-Based Chemotherapy for Advanced Non-Small-Cell Lung Cancer:Meta-Analysis of Randomized Trials. J Clin Oncol. 2006;24(3):419-430.

16. Dong J, Su SY, Wang MY, et al. Shenqi fuzheng, an injection concocted from Chinese medicinal herbs, combined with platinum-based chemotherapy for advanced non-small cell lung cancer:a systematic review. J Exp Clin Cancer Res. 2010;29:137.

17. Richardson MA, Sanders T, Palmer JL, et al. Complementary/Alternative Medicine Use in a Comprehensive Cancer Center and the Implications for Oncology. J Clin Oncol. 2000;18(13):2505-2514.

18. Block KI, Gyllenhaal C. Clinical Corner:herb-Drug Interactions in Cancer Chemotherapy:Theoretical Concerns Regarding Drug Metabolizing Enzymes. Integr Cancer Ther. 2002;1(1):83-89.

19. Hu Z, Yang X, Ho PCL, et al. Herb-Drug Interactions:a literature review. Drugs. 2005;65(9):1239-1282.

20. Kennedy DA, Seely D. Clinically based evidence of drug-herb interactions:a systematic review. Expert Opin Drug Saf. 2010;9(1):79124.

21. Cui Y, Shu XO, Gao Y, et al. Use of complementary and alternative medicine by Chinese women with breast cancer. Breast Cancer Res Treat. 2004;85(3):263-270.

22. Chen Z, Gu K, Zheng Y, et al. The use of complementary and alternative medicine among Chinese women with breast cancer. J Altern Complement Med. 2008;14(8):1049-55.

23. MeSH search 28 Aug 2014:(contradiction[All Fields] AND herbs[All Fields]) AND ("drug therapy"[Subheading] OR ("drug"[All Fields] AND "therapy"[All Fields]) OR "drug therapy”[All Fields] OR "chemotherapy"[All Fields] OR "drug therapy"[MeSH Terms] OR ("drug”[All Fields] AND “therapy”[All Fields]) OR "chemotherapy”[All Fields]) produced no results.

24. Broom A, Adams J. Oncology clinicians' accounts of discussing complementary and alternative medicine with their patients. Health (London). 2009;13(3):317-336.

25. Lewith GT, Breen A, Filshie J, et al. Complementary medicine:evidence base, competence to practice and regulation. Clin Med. 2003;3(3):235240

26. Cassileth BR, Vickers AJ. High Prevalence of Complementary and Alternative Medicine Use Among Cancer Patients:Implications for Research and Clinical Care. J Clin Oncol. 2005;23(12):2590-2592.

27. Janes KA, Wang CC. Bringing systems biology to cancer, immunology and infectious disease. Genome Biol. 2014;15(7):407.
28. Cho WCS. An Omics Perspective on Cancer Research. in Omics Perspective on Cancer Research. Cho WCS, 1st edn. USA: Springer. Dordrecht; pp 1-267.

29. Fardet A, Rock E. Toward a new philosophy of preventive nutrition:from a reductionist to a holistic paradigm to improve nutritional recommendations. Adv Nutr. 2014;5(4):430-446.

30. Henry SG. Recognizing tacit knowledge in medical epistemology. Theor Med Bioeth. 2006;27(3):187-213.

31. Devisch I, Murray SJ. 'We hold these truths to be selfevident':deconstructing 'evidence-based' medical practice. J Eval Clin Pract. 2009; 15(6):950-954.

32. Chaulk CP, Kazandjian VA. Moving beyond randomized controlled trials. Am J Public Health. 2004;94(9):1476.

33. Henry SG, Zaner RM, Dittus RS. Viewpoint:moving beyond evidencebased medicine. Acad Med. 2007;82(3):292-297.

34. Kazandjian VA, Matthes N, Wicker KG. Are performance indicators generic? The international experience of the Quality Indicator Project. $J$ Eval Clin Pract. 2003;9(2):265-276.

35. Kazandjian VA, Lied TR. Healthcare Performance Measurement:Systems Design And Evaluation. Milwaukee, Wisc: ASQ Quality Press; 1999.

36. Bensing J. Bridging the gap. The separate worlds of evidencebased medicine and patient-centered medicine. Patient Educ Couns. 2005;39(1):17-25.

37. Epstein RM, Franks P, Fiscella K, et al. Measuring patient-centered communication in Patient-Physician consultations:Theoretical and practical issues. Soc Sci Med. 2005;61(7):1516-1528.

38. Mead N, Bower P. Patient-centredness:a conceptual framework and review of the empirical literature. Soc Sci Med. 2000;51(7):1087-1110.

39. London L, Himonga C, Fick N, et al. Social solidarity and the right to health:essential elements for people-centred health systems. Health Policy Plan. 2014.

40. Zaslawski C. Clinical reasoning in traditional Chinese medicine:implications for clinical research. Clinical Acupuncture and Oriental Medicine. 2003;4(2-3):94-101.

41. Jiang M, Zhang C, Zheng G, et al. Traditional Chinese Medicine Zheng in the Era of Evidence-Based Medicine:A Literature Analysis. Evid Based Complement Alternat Med. 2012; 2012

42. Feng Y, Wu Z, Zhou X, et al. Knowledge discovery in traditional Chinese medicine:state of the art and perspectives. Artif Intell Med. 2006;38(3):219-236.

43. Herman PM, Szczurko O, Cooley K, et al. Cost-effectiveness of naturopathic care for chronic low back pain. Altern Ther Health Med. 2008;14(2):32-39.

44. Zhang X, Wang W, Xiao K, et al. Translational Medicine: Applications of Omics for Drug Target Discovery and Validation. in Omics Perspective on Cancer Research. In: Cho WCS, Editor. Dordrecht: Springer; 2010.

45. Dai J, Fang J, Sun S, et al. ZHENG-Omics Application in ZHENG Classification and Treatment:Chinese Personalized Medicine. Evid Based Complement Alternat Med. 2013

46. Wang $\mathrm{P}$, Chen Z. Traditional Chinese medicine ZHENG and Omics convergence:a systems approach to post-genomics medicine in a global world. OMICS. 2013;17(9):451-459. 